*For correspondence: aleksej.stupnikov@phystech.edu (FMS); ju.medvedeva@gmail.com (FS)

\title{
Hobotnica: exploring molecular signature quality
}

3 Alexey Stupnikov ${ }^{1,6^{*}}$, Alexey Sizykh ${ }^{1}$, Alexander Favorov ${ }^{2,5}$, Bahman Afsari ${ }^{2}$, Sarah 4 Wheelan $^{2}$, Luigi Marchionni ${ }^{3}$, Yulia A. Medvedeva ${ }^{1,4,6^{*}}$

$5 \quad{ }^{1}$ Moscow Institute of Physics and Technology, Moscow, Russia; ${ }^{2}$ Johns Hopkins 6 University, Baltimore, USA; ${ }^{3}$ Weill Cornell Medicine, New York, USA; ${ }^{4}$ Center of Biotechnology RAS, Moscow, Russia; ${ }^{5}$ Vavilov Institute for General Genetics RAS, Moscow, Russia; ${ }^{6}$ National Medical Research Center for Endocrinology, Moscow, Russia

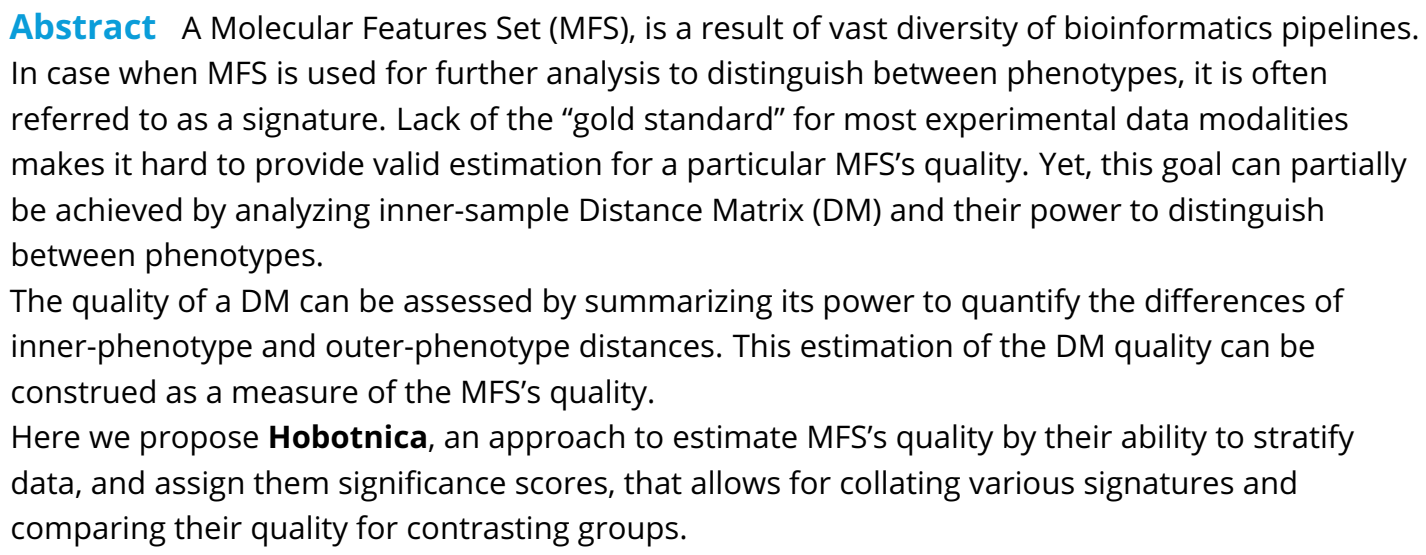

\section{Introduction}

A signature based on a predefined Molecular Features Set (MFS), which is designed to distinguish biological conditions or phenotypes from each other - is one of major concepts of bioinformatics and precision medicine. In this context, signatures typically originate from MFS from contrasting experimental data of two or more sample groups, which differ phenotypically. These MFS incorporate information on the differences between the groups. The nature of the MFS depends on the modality of the original data. For instance, the MFS provided by the Differential Gene Expression approach is a list of Differentially Expressed Genes (DEG); Differential Methylation analysis provides Differentially Methylated Cytosines or Regions (DMC and DMR) as MFS, etc.

A significant number of mutational, expression and methylation-based signatures have recently been published and they are actively used in Research and Transnational Medicine. Examples of expression-based signatures involve genesets for clinical prognosis (e.g. PAM50 (Parker et al. (2009)), MammaPrint (Cardoso et al. (2016)) for Breast Cancer), for pathways and gene enrichment analysis (e.g. MsigDB collections (Subramanian et al. (2005))), for drug re-purposing (e.g. LINCS project(Liu et al. (2015))).

Direct quality assessment for MFS is currently hardly possible, since there are no 'gold standard' datasets where active Molecular Features are explicitly known. In this manuscript, we propose a novel approach - Hobotnica - that allows for measurement of MFS quality by addressing the key property of the signature, namely, its quality for data stratification.

Hobotnica leverages the quality of Distance Matrices obtained from any source in order to as- 
sess quality of the MFS from any data modality compared to a random MFS. In this study, we demonstrate its application on transcriptomic signatures.

\section{Results \\ Approach}

The Hobotnica approach is as follows: For a given data set $W$ and a given Molecular Features Set $(S)$ we derive the inter-sample distance matrix $(D M(S, W))$. Then we assess the quality of $D M$ (and, thus, of $S$ ) with a summarizing function $(\alpha(D M(S))=\alpha(D M(S), Y)$ or by abuse of notation $\alpha(D M(S)))$ where $(Y)$ represents the labels of samples.

We desire the function $\alpha$ to gauge if the inner-class samples are closer to each other than to outer-class samples. If no difference exists from one class to another, $\alpha$ must be close to zero and as the difference grows, $\alpha$ grows. In ideal case of a perfect separation, $\alpha$ reaches its maximum at 1:

- $\alpha \in[0,1]$

- $\alpha \rightarrow 1 \Leftrightarrow$ High groups stratification quality

- $\alpha \rightarrow 0 \Leftrightarrow$ Low groups stratification quality

Under the Null hypothesis of Hobotnica $\left(\left(H_{0}\right)\right)$, no significant difference exists between $\alpha(S)$ and the $\alpha$ of an equal-sized general random set. On the contrary, the Alternative $\left(H_{A}\right)$ hypothesizes that $S$ generates higher $\alpha$ than most random $S^{\prime}$ of the same size. To estimate a Null distribution for Hobotnica's $\alpha$, we applied a permutation test. As our default options, we use Kendall distance as the distance measure and Mann-Whitney-Wilcoxon test as the summarizing function.

\section{Validation}

To validate our approach in the first case study we extracted RNA-seq expression dataset for Prostate Cancer from TCGA on counts level(Rahman et al. (2015)). As Molecular Feature Sets we recruited C2 collection of molecular signatures from MSigDB (Subramanian et al. (2005), Liberzon et al. (2011)) that contains a number of Prostate-related genesets. For the second case study we took PAM50 molecular signature, designed for various Breast Cancer types classification, and applied it to several datasets (Marusyk et al. (2016))(Daemen et al. (2013))(Costello et al. (2014))(Rahman et al. (2015))(Varley et al. (2014)). In both cases, the counts were normalised to cpm. For each geneset $\mathrm{H}$-score and its $\mathrm{p}$-value with $\mathrm{BH}$ correction were computed.

Prostate-related $\mathrm{C} 2$ genesets clearly demonstrated highest values of $\mathrm{H}$-score and sufficient statistical significance(Fig.1.A), as well as data stratification (Fig.1.B), which is expected for Prostate Cancer vs Control contrast. Genesets not attributed to Prostate Cancer related processes did not achieve statistical significant p-values. (Table1).

PAM50 signature evidently separates samples in for GSE48216 dataset (Fig.1.C). H-scores for random genesets for the same dataset are significantly lower than an H-score for PAM50 (Fig.1.D). Clearly, PAM50 signature demonstrates high quality of stratification for the samples of various Breast Cancer datasets with high $\mathrm{H}$-score values and statistically significant p-values (Table 2).

Thus, in the first case study, Prostate Cancer related genesets from C2 collection, when applied to Prostate Cancer dataset, delivered highest $\mathrm{H}$-scores and most significant and $\mathrm{p}$-values proved to demonstrate best scores and performance. Likewise, in the second case study, PAM50 expression signature applied to several heterogeneous Breast Cancer datasets delivered high $\mathrm{H}$-score values along with significant scores of $p$-values.

\section{Application}

An important question that researches often face is establishing the optimal size of the retrieved signature. The exact number of genes to be retrieved from the set of all significant genes is an important parameter that is essential for signature's application. To explore the optimal size of DE 
bioRxiv preprint doi: https://doi.org/10.1101/2021.09.12.459931; this version posted September 15, 2021. The copyright holder for this preprint (which was not certified by peer review) is the author/funder. All rights reserved. No reuse allowed without permission.

Table 1. 10 C2-CGP Gene Signatures with highest H-scores

\begin{tabular}{lrr}
\hline Signature & H-score & p-value \\
\hline TOMLINS_PROSTATE_CANCER & 0.795 & 0.025 \\
WALLACE_PROSTATE_CANCER & 0.747 & 0.025 \\
OUYANG_PROSTATE_CANCER_PROGRESSION & 0.745 & 0.025 \\
LIU_PROSTATE_CANCER & 0.735 & 0.025 \\
PIEPOLI_LGI1_TARGETS & 0.724 & 0.059 \\
SMID_BREAST_CANCER_RELAPSE_IN_LIVER & 0.712 & 0.164 \\
TIMOFEEVA_GROWTH_STRESS_VIA_STAT1 & 0.708 & 0.240 \\
GENTILE_UV_LOW_DOSE & 0.705 & 0.308 \\
JOHANSSON_BRAIN_CANCER_EARLY_VS_LATE & 0.701 & 0.377 \\
HOWLIN_CITED1_TARGETS_1 & 0.700 & 0.377 \\
\hline
\end{tabular}

Table 2. PAM50 results

\begin{tabular}{lcccr}
\hline GEO Accession & Sample size & Groups in dataset & H-score & p-value \\
\hline GSE58135 & 168 & 6 & 0.772 & $7 \mathrm{e}-4$ \\
GSE62944 & 1067 & 5 & 0.8892 & 0.0003 \\
GSE48216 & 46 & 3 & 0.8567 & 0.0003 \\
GSE80333 & 10 & 3 & 0.9765 & 0.0003 \\
\hline
\end{tabular}
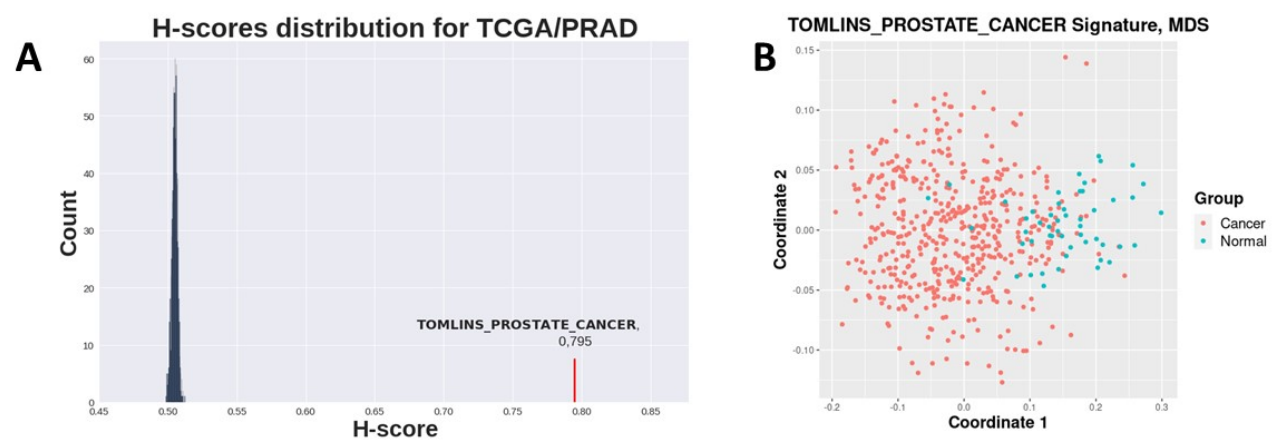

C
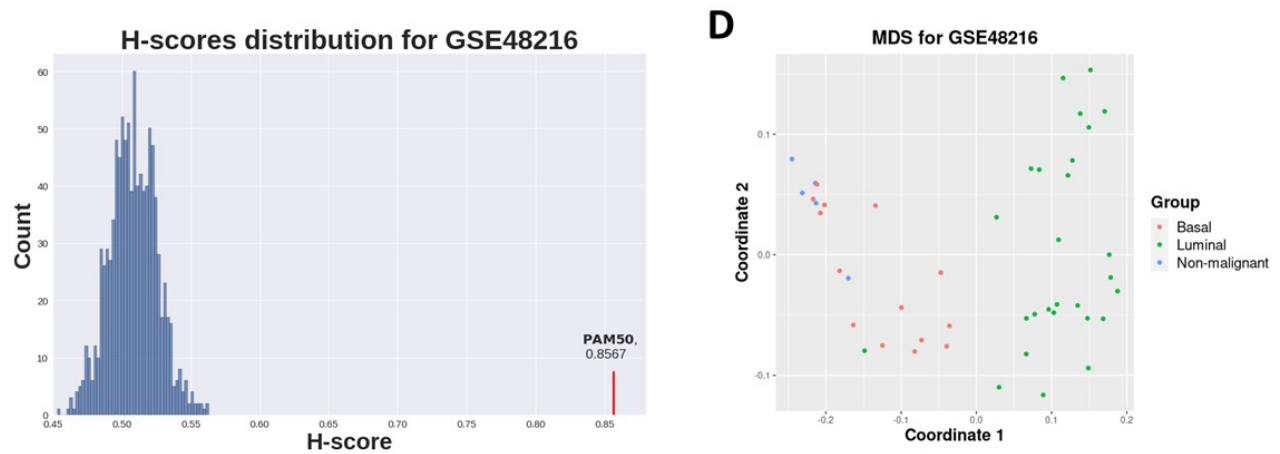

Figure 1. A : Distribution of H-scores for random genesets (blue) on TCGA Prostate Cancer vs Normal dataset (see Tab.1) and Tomlins prostate geneset H-score (red). B: MDS for TCGA Prostate demonstrates samples separation with Tomlins geneset. C: Distribution of H-scores for random genesets (blue) on GSE48216 Breast Cancer dataset (see Tab.2) and PAM50 geneset H-score (red). D: MDS for GSE48216 Breast Cancer dataset samples separation with PAM50 geneset.

signature we performed Hobotnica analysis for top DE p-value ordered gene signatures of various lengths. For the reference we performed DGE analysis for Breast Cancer vs Control TCGA dataset 

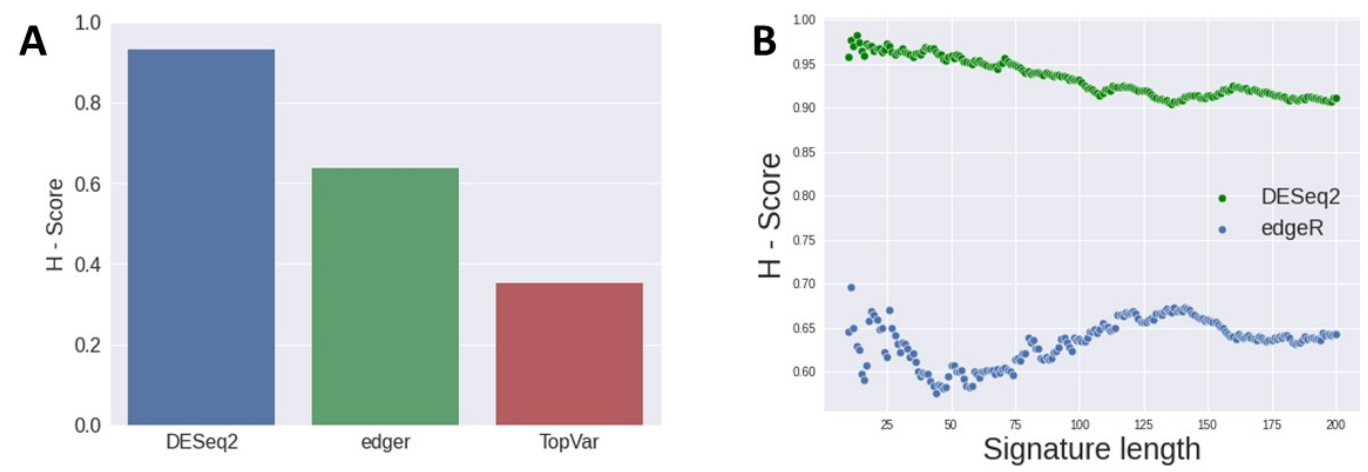

Figure 2. A: H-scores delivered by top 100 gene signatures from various DE models applied to TCGA Breast Cancer data. B: Change of $\mathrm{H}$-score with the length of gene signature derived from DESeq2 and edgeR models

(Rahman et al. (2015)) with DESeq2 (Love et al. (2014)) and edgeR (McCarthy et al. (2012)). Top 100 genes for each method were retrieved, as well as genes with highest variance in expression. $\mathrm{H}$-scores for every signature then were computed (Fig.2.A.). For this dataset DESeq2 provided a signature with the highest quality score. Then, we calculated $\mathrm{H}$-score for signatures of various lengths (Fig.2.B.) Surprisingly, the signature quality is non-monotonously dependant on the signature length, i.e. number of genes in the signature. The pattern also varies for DE models. Additionally, for the best-performing model, DESeq2, the a signature quality is generally declining with the length. Thus, increasing number of genes in a signature may not improve its quality, and an optimal gene signature length for the DE analysis result may be established: DESeq2 signature reaches its maximum $\mathrm{H}$-score at 13 genes and edge $\mathrm{R}$ at 11 in this case.

\section{Discussion}

Hobotnica is designed to quantitatively evaluate Molecular Feature Set's quality by their ability for data stratification from their inter-sample distance matrices, and to assess the statistical significance of the results. We demonstrated that Hobotnica can efficiently estimate the quality of a Molecular Signature in the context of Expression data.

Suggested method can be used to evaluate Molecular Feature sets of various nature: retrieved in DGE, Differential Methylation analysis, Mutation/SNV calling or Pathways analysis, as well as data modalities from other types of Differential Problem. In addition, assessing $\mathrm{H}$-score values for various lengths of the same set or signature will help with its structure optimization, which may be especially important in clinical applications.

Hobotnica is available as an R package at https://github.com/lab-medvedeva/Hobotnica-main

\section{Methods and Materials}

\section{Problem formalization}

If a Molecular Feature Set $(S)$, that presumably incorporates information on the contrast between groups of samples with known samples annotation $Y$ in Data $D$ is in place $(H: S)$, we can compute Distance Matrix between samples $D M(f(S \mid D) \rightarrow D M)$ and then introduce a measure $\alpha$ $(g(D M \mid Y) \rightarrow \alpha)$ of signature quality for Data $D$ stratification.

$$
\begin{gathered}
H: S \\
f(S \mid D) \rightarrow D M \\
g(D M \mid Y) \rightarrow \alpha
\end{gathered}
$$


118

119

120

121

122

123

124

125

126

127

128

129

130

131

132

133

134

135

136

137

138

139

140

141

142

143

144

145

146

147

148

149

150

151

152

153

154

155

156

157

When instead of a single $G S$ a set of hypotheses $\left\{H_{1}: G S_{1}, H_{2}: G S_{2}, \ldots, H_{n}: G S_{n}\right\}$ is in place, for each Gene Signature $G S_{i}$ corresponding Distance Matrix $D M_{i}$ can be generated, and than, in turn, particular value of the measure $\alpha_{i}$ :

$$
\left\{\begin{array} { l } 
{ H _ { 1 } : S _ { 1 } } \\
{ H _ { 2 } : S _ { 2 } } \\
{ \cdots } \\
{ H _ { n } : S _ { n } }
\end{array} \rightarrow \left\{\begin{array} { l } 
{ f ( S _ { 1 } | D ) \rightarrow D M _ { 1 } } \\
{ f ( S _ { 2 } | D ) \rightarrow D M _ { 2 } } \\
{ \cdots } \\
{ ( S _ { n } | D ) \rightarrow D M _ { n } }
\end{array} \quad \rightarrow \left\{\begin{array}{l}
g\left(D M_{1} \mid A\right) \rightarrow \alpha_{1} \\
g\left(D M_{2} \mid A\right) \rightarrow \alpha_{2} \\
\cdots \\
g\left(D M_{n} \mid A\right) \rightarrow \alpha_{n}
\end{array} .\right.\right.\right.
$$

Thus, for every MFS $S_{i}$ from set of hypotheses $\left\{H_{1}: S_{1}, H_{2}: S_{2}, \ldots, H_{n}: S_{n}\right\}$ H-score $\alpha_{i}$ may be computed, resulting in a set $\left\langle\alpha_{1}, \alpha_{2}, \ldots \alpha_{n},\right\rangle$. Comparing $\alpha$ values allows for corresponding Feature Sets qualities ranking and selecting the most informative Signatures for the Data $D$.

To assess statistical significance of each obtained $\mathrm{H}$-score $\alpha_{i}$ we compute empirical $p$-value via generating a distribution of $\mathrm{H}$-scores for set of random MFS.

\section{Availability}

We implemented Hobotnica as an R package available at https://github.com/lab-medvedeva/Hobotnicamainhttps://github.com/lab-medvedeva/Hobotnica-main. It contains an implementation of the Hobotnica measure, statistical analysis for significance, and several auxiliary functions for visualizing results and parallel processing.

\section{Acknowledgements}

We thank Frank Emmert-Streib, Leslie Cope and Elana Fertig for fruitful discussions. The study was supported by Ministry of Science and Higher Education of the Russian Federation (agreement no. 075-15-2020-899) and by the NIH grants R01DE027809 and P30CA006973.

\section{References}

Cardoso F, van't Veer LJ, Bogaerts J, Slaets L, Viale G, Delaloge S, Pierga JY, Brain E, Causeret S, DeLorenzi M, et al. 70-gene signature as an aid to treatment decisions in early-stage breast cancer. New England Journal of Medicine. 2016; 375(8):717-729.

Costello JC, Heiser LM, Georgii E, Gönen M, Menden MP, Wang NJ, Bansal M, Hintsanen P, Khan SA, Mpindi JP, et al. A community effort to assess and improve drug sensitivity prediction algorithms. Nature biotechnology. 2014; 32(12):1202-1212.

Daemen A, Griffith OL, Heiser LM, Wang NJ, Enache OM, Sanborn Z, Pepin F, Durinck S, Korkola JE, Griffith M, et al. Modeling precision treatment of breast cancer. Genome biology. 2013; 14(10):1-14.

Liberzon A, Subramanian A, Pinchback R, Thorvaldsdóttir H, Tamayo P, Mesirov JP. Molecular signatures database (MSigDB) 3.0. Bioinformatics. 2011 05; 27(12):1739-1740. https://doi.org/10.1093/bioinformatics/ btr260, doi: 10.1093/bioinformatics/btr260.

Liu C, Su J, Yang F, Wei K, Ma J, Zhou X. Compound signature detection on LINCS L1000 big data. Molecular BioSystems. 2015; 11(3):714-722.

Love MI, Huber W, Anders S. Moderated estimation of fold change and dispersion for RNA-seq data with DESeq2. Genome biology. 2014; 15(12):1-21.

Marusyk A, Tabassum DP, Janiszewska M, Place AE, Trinh A, Rozhok Al, Pyne S, Guerriero JL, Shu S, Ekram M, et al. Spatial proximity to fibroblasts impacts molecular features and therapeutic sensitivity of breast cancer cells influencing clinical outcomes. Cancer research. 2016; 76(22):6495-6506.

McCarthy DJ, Chen Y, Smyth GK. Differential expression analysis of multifactor RNA-Seq experiments with respect to biological variation. Nucleic Acids Research. 2012 01; 40(10):4288-4297. doi: 10.1093/nar/gks042.

Parker JS, Mullins M, Cheang MC, Leung S, Voduc D, Vickery T, Davies S, Fauron C, He X, Hu Z, et al. Supervised risk predictor of breast cancer based on intrinsic subtypes. Journal of clinical oncology. 2009; 27(8):1160. 
bioRxiv preprint doi: https://doi.org/10.1101/2021.09.12.459931; this version posted September 15, 2021. The copyright holder for this preprint (which was not certified by peer review) is the author/funder. All rights reserved. No reuse allowed without permission.

158 Rahman M, Jackson LK, Johnson WE, Li DY, Bild AH, Piccolo SR. Alternative preprocessing of RNA-Sequencing 159 data in The Cancer Genome Atlas leads to improved analysis results. Bioinformatics. 2015; 31(22):3666-3672.

161

162

163

Subramanian A, Tamayo P, Mootha VK, Mukherjee S, Ebert BL, Gillette MA, Paulovich A, Pomeroy SL, Golub TR, Lander ES, et al. Gene set enrichment analysis: a knowledge-based approach for interpreting genome-wide expression profiles. Proceedings of the National Academy of Sciences. 2005; 102(43):15545-15550.

Varley KE, Gertz J, Roberts BS, Davis NS, Bowling KM, Kirby MK, Nesmith AS, Oliver PG, Grizzle WE, Forero A, 164 et al. Recurrent read-through fusion transcripts in breast cancer. Breast cancer research and treatment. $165 \quad 2014 ; 146(2): 287-297$. 